\title{
Robust Multi-Period Portfolio Model Based on Prospect Theory and ALMV-PSO Algorithm
}

\author{
Jiahe Liu* $\quad$ Xiu Jin ${ }^{\dagger} \quad$ Tianyang Wang ${ }^{\ddagger} \quad$ Ying Yuan ${ }^{\S}$
}

April 16, 2015

\begin{abstract}
The studies of behavioral finance show that the cognitive bias plays an important role in investors' decision-making process. In this paper, based on the robust theory and prospect theory, a robust multi-period portfolio considering investors' behavioral factors is constructed, which features the reference dependence, loss aversion and diminishing sensitivity. To solve the proposed portfolio model, an improved particle swarm optimization (PSO) algorithm is developed, which incorporates the two-stage initialization strategy, the improved stochastic ranking approach, the aging leader and the multi-frequency vibrational mutation operator. We illustrate the robust model with real market data and show its effectiveness based on the performance of the proposed algorithm. The results show that the proposed algorithm is successful in solving the constrained multi-period portfolio model and the proposed portfolio model provides an effective tool for a real multi-period investment.
\end{abstract}

Keywords: Finance; Portfolio selection; Prospect theory; Robust optimization; Multiperiod portfolio; Particle swarm optimization

\footnotetext{
*Department of Finance, Northeastern University, China. Email: jhliu.research@gmail.com ${ }^{\dagger}$ Department of Finance, Northeastern University, China. Email: xjin@mail.neu.edu.cn

${ }^{\ddagger}$ Department of Finance \& Real Estate, Colorado State University. Email: tianyang.wang@business.colostate.edu $\S$ Department of Finance, Northeastern University, China. Email: yyuan@mail.neu.edu.cn
} 


\section{Robust Multi-Period Portfolio Model Based on Prospect Theory and ALMV-PSO Algorithm}

\section{Introduction}

Portfolio optimization as an effective wealth allocation and risk management tool has attracted a lot attention from both academics and practitioners. The mean-variance model formulated by Markowitz (1952) lays the foundation of modern portfolio selection theory. The main goal of the mean-variance model is to optimally allocate wealth by considering the trade-off between risk and return. Recently, substantial effort has been devoted to extending the portfolio theory based on Markowitz's framework, including the multi-period portfolio problem with a kernel-based control policy (Takano and Gotoh, 2014), the mean-variance portfolio based on a stochastic benchmark (Bernard and Vanduffel, 2014), the portfolio management with financial ratios and technical indicators (Silva et al. 2015), and the multi-objective portfolio considering the dependence structure of asset returns (Babaei et al., 2015). We refer the interested readers to Markowitz (2014) for a detailed discussion about a half-century of research on mean-variance approximations to the expected utility.

In the framework of the mean-variance model, security returns are regarded as random variables, and expected returns are denoted as investment returns for securities. However, the accurate distributions of security returns are difficult to obtain through historical data. Moreover, the meanvariance model is highly sensitive to expected returns: a small perturbation in expected returns may lead to a large variation in the optimum portfolio allocation (cf. Best and Grauer, 1991; Black and Litterman, 1992). Therefore, it is more reasonable to regard the distributions of security returns as interval random uncertainty sets (cf. Chen et al., 2011; Moon and Yao, 2011; Chen and Kwon, 2012), which reflect investors' uncertainty about parameters and therefore reduce the sensitivity of portfolio optimization models on expected returns.

We join a substantial list of researchers that examine the robust portfolio model. To remedy the problem of parameter uncertainty, Soyster (1973) first employed the concept of robust optimization to solve an inexact linear programming problem. In the framework of Soyster (1973), the values of parameters are defined as uncertainty sets, and decision-makers make decisions under the worst- 
case scenario within the sets. Bertsimas and Sim (2004) developed a new robust approach for uncertain linear optimization problems with the Soyster (1973)'s framework as a special case, which provides flexibility of selecting the degree of conservatism of the solution. With the development of the robust optimization theory, the robust optimization method has been widely used in investment decision-making. Based on the robust optimization approach of Bertsimas and Sim (2004), several new robust models have been proposed, such as the robust mean absolute deviation portfolio model (Moon and Yao, 2011), the robust index tracking portfolio model (Chen and Kwon, 2012), the robust multi-period portfolio model (Gharakhani and Sadjadi, 2011) and the robust multi-objective R\&D project portfolio model (Hassanzadeh et al., 2014). In addition, using a robust regressionbased approach, Maillet et al. (2015) introduced a robust global minimum variance portfolio model to mitigate the impact of parameter uncertainty. By integrating a robust optimization approach with a mixture distribution modeling scheme, Zhu et al. (2014) developed a portfolio selection framework with a feature of double robustness in both return distribution modeling and portfolio optimization. Ai, Brocket and Wang (2013) examine the corporate optimal portfolio under the Dynamic enterprise risk management framework.

While most of robust portfolios are proposed under the hypothesis that investors are perfectly rational beings, this hypothesis does not always hold in real life. The studies of behavioral finance have found that the axioms of rationality are violated across a range of financial decision-making situations (e.g. Tiwana et al., 2007; Heiman et al., 2015), and the cognitive biases of investors have great influence on the decision-making processes (e.g. Kahneman and Tversky, 1979; Liu et al., 2014). Prospect theory proposed by Kahneman and Tversky (1979) provides both the descriptive theory for individual's actual decision-making behavior under uncertainty and the theoretical foundation of optimal portfolios that take into account the behavioral factors of investors.

Under the mean-variance framework, portfolio models based on prospect theory have been developed in recent years. Shefrin and Statman (2000) developed a behavioral portfolio theory based on the foundation of SP/A theory and prospect theory. Since the seminal work of Shefrin and Statman (2000), there is a vast body of literature considering the behavioral portfolio theory (e.g. Das and Statman, 2013; Jiang et al., 2013; Magron, 2014; Hoffmann and Shefrin, 2014). In addition, Blake et al. (2013) built an asset allocation model which uses the prospect theory value function to reflect investors' behavioral traits in the defined contribution pension planning. 
More recently, Guo (2014) incorporated the cumulative prospect theory into the insurance portfolio model, where the preference of decision-maker is defined by an S-shaped utility function. While these studies produce important pieces of information relating to the nature of optimal portfolios subject to a range of psychological factors, they do not make an attempt at robust portfolios.

The purpose of this study is to incorporate the impact of investors' behavioral factors into the robust multi-period portfolio model. The key differences between our paper and existing literatures are as follows. First, existing robust portfolio models seldom consider investors' behavioral factors (e.g. Hassanzadeh et al., 2014; Maillet et al., 2015; Zhu et al., 2014). We formulate a robust multi-period portfolio model featuring the reference dependence, loss aversion and diminishing sensitivity, where the loss aversion parameters are dynamically updated based on prospect theory. Moreover, to control the conservatism level of the robust solution intuitively, the most violated probability is introduced, which reflects investors' safety requirement. Second, we introduce a novel particle swarm optimization (PSO) algorithm with an aging leader and multi-frequency vibration (ALMV-PSO) for the robust multi-period portfolio problem. In the ALMV-PSO algorithm, an aging leader and a multi-frequency vibrational mutation operator are employed, which can reduce the probability of being trapped into local optimal. Additionally, we also design a twostage initialization strategy and an improved stochastic ranking approach for PSO. The two-stage initialization strategy guarantees that all of the initial particles are in a feasible region and with a high level of diversity. The improved stochastic ranking approach balances between the objective function value and the constraint violation function value for the constrained portfolio problem.

The rest of this paper is organized as follows. Section 2 constructs a robust multi-period portfolio model featuring the reference dependence, loss aversion and diminishing sensitivity, where loss aversion parameters are dynamically updated. Section 3 proposes an ALMV-PSO algorithm to solve the proposed robust model. In Section 4, a real market data example is used to illustrate the portfolio model and check the effectiveness of the ALMV-PSO algorithm. Finally, we present conclusions of the paper and directions for further research in Section 5. 


\section{Robust Multi-period Portfolio Model Based on Prospect Theory}

The studies of behavioral finance show that investors could have numerous cognitive biases (e.g. mental accounting, loss aversion, etc.), which play important roles in decision-making process (cf. Shefrin and Statman, 2000; Barberis and Huang, 2001). While lots of current literatures focus on the subjects of the behavioral portfolio model (e.g. Das and Statman, 2013; Jiang et al., 2013; Guo, 2014) and the robust portfolio model (e.g. Hassanzadeh et al., 2014; Zhu et al., 2014; Maillet et al., 2015), these studies have been in separate fashions, making a simplified assumption that neglects the joint impact of investors' irrationality and incomplete information of future returns. In this paper, we study the robust multi-period portfolio model with behavioral factors, which not only addresses the parameter uncertainty, but also takes investors' reference dependence, loss aversion and diminishing sensitivity into consideration.

\subsection{Problem Description}

Consider that there are one riskless asset $a_{0}$ and $n$ risky assets $\left\{a_{1}, \ldots, a_{n}\right\}$ in security market for trading. An investor wants to make a multi-period investment strategy, where the investment duration is divided into $T$ periods. Suppose that the investor holds a portfolio $\mathbf{X}(t)=$ $\left[x_{0, t}, x_{1, t}, \ldots, x_{n, t}\right]^{\top}$ at time $t$, where $x_{0, t}$ denotes the wealth of riskless asset $a_{0}$ at time $t$, and $x_{i, t}$ denotes the wealth of risky asset $a_{i}$ at time $t, i=1, \ldots, n, t=0, \ldots, T$.

The investor could dynamically adjust the portfolio at the end of each period based on the realized return and updated information about the security market. Let $\Delta \mathbf{X}(t)=\left[\Delta x_{0, t}, \Delta x_{1, t}, \ldots\right.$, $\left.\Delta x_{n, t}\right]^{\top}$ be the adjustment of the portfolio at time $t$, where $\Delta x_{i, t}>0$ means the wealth of asset $a_{i}$ is increased at time $t$, and $\Delta x_{i, t}<0$ means the wealth of asset $a_{i}$ is decreased at time $t$, $i=0,1, \ldots, n, t=0, \ldots, T-1$. Thus we obtain the adjusted portfolio $\mathbf{X}^{+}(t)=\left[x_{0, t}^{+}, x_{1, t}^{+}, \ldots, x_{n, t}^{+}\right]^{\top}$ after the adjustment according to $\Delta \mathbf{X}(t)$ at time $t$, where $\mathbf{X}^{+}(t)=\mathbf{X}(t)+\Delta \mathbf{X}(t), t=0, \ldots, T-1$. Following Calafiore (2008), the multi-period investment procedure is shown in Fig. 1.

[Insert Figure 1 Here]

Let $r_{0, t}$ and $r_{i, t}$ be the return of riskless asset $a_{0}$ and risky asset $a_{i}$ at period $t$ respectively, 
$i=1, \ldots, n, t=1, \ldots, T$. Then the wealth of asset $a_{i}$ at time $t$ is given as

$$
x_{i, t}=\left(r_{i, t}+1\right) x_{i, t-1}^{+}, i=0,1, \ldots, n, t=1, \ldots, T
$$

Using the recursive relationship in the multi-period investment, we can rewrite Eq. (1) as

$$
x_{i, t}=g_{i}(1, t) x_{i, 0}+\sum_{j=1}^{t} g_{i}(j, t) \Delta x_{i, j-1}, i=0,1, \ldots, n, t=1, \ldots, T
$$

where $g_{i}(j, t)$ denotes the cumulative return of asset $a_{i}$ from period $j$ to period $t, g_{i}(j, t)=\left(r_{i, t}+\right.$ 1) $\left(r_{i, t-1}+1\right) \cdots\left(r_{i, j}+1\right), g_{i}(t, t)=r_{i, t}+1$.

By Eq. (2), the multi-period portfolio wealth at time $t$ is given by

$$
W_{t}=\sum_{i=0}^{n} x_{i, t}=\sum_{i=0}^{n} \sum_{j=1}^{t} g_{i}(j, t) \xi_{i, j-1}, t=1, \ldots, T
$$

where $\xi_{i, 0}=x_{i, 0}+\Delta x_{i, 0}, \xi_{i, j}=\Delta x_{i, j}, i=0,1, \ldots, n, j=1, \ldots, T-1$.

\subsection{Robust Optimization Approach}

If the investor exactly knew the future return of each asset, this would be a classic deterministic multi-period portfolio optimization problem. In practice, since it is difficult to estimate asset returns exactly, the deterministic assumption of the portfolio optimization problem is invalid. Robust optimization has emerged as a leading methodology for addressing the uncertainty in optimization problems $1^{1}$ In this paper, we adopt the robust optimization framework of Bertsimas and Sim (2004) that has been widely used in decision-making for uncertain optimization problems (e.g. Chen and Kwon, 2012; Hassanzadeh et al., 2014). To balance the trade-off between the optimality of the solution and its robustness to return perturbation, Bertsimas and Sim (2004)'s robust optimization framework provides flexibility of the degree of conservatism of the solution. 2

\footnotetext{
${ }^{1}$ The multi-period stochastic programming model has also been proposed. At each stage, it applies the policy that is optimal when maximizing the expected value of the risk adjusted returns. The computational results of stochastic programming algorithms have been shown to violate the original constrains with certain probability and to be outperformed by the robust optimization both in terms of efficiency and optimal strategy selection (cf. BenTal and Nemirovski, 1998, 1999; Bertsimas and Pachamanova, 2008). In this paper, we will focus on the robust optimization approach for multi-period portfolio selection.

${ }^{2}$ See Bertsimas and Sim (2004) and Gabrel et al. (2014) for a detailed discussion about different robust optimization methods.
} 
We will assume the cumulative return $g_{i}(j, t)$ takes values in the interval $\left[\bar{g}_{i}(j, t)-\hat{g}_{i}(j, t), \bar{g}_{i}(j, t)+\right.$ $\left.\hat{g}_{i}(j, t)\right]$, where $\bar{g}_{i}(j, t)$ denotes the nominal value, and $\hat{g}_{i}(j, t)$ denotes the half-interval width of $\left.g_{i}(j, t), i=0,1, \ldots, n, j=1, \ldots, t, t=1, \ldots, T\right]^{3}$ Suppose that the cumulative return of riskless asset is exactly known, while the cumulative return of risky asset is uncertain, i.e. $\hat{g}_{0}(j, t)=0$, $\hat{g}_{i}(j, t)>0, i=1, \ldots, n, j=1, \ldots, t, t=1, \ldots, T$, which is in line with our practice. Thus, based on Eq. (3), the portfolio wealth $W_{t}$ contains $n \cdot t$ uncertain variables.

The goal of robust optimization is to find a solution which is feasible for all possible data realizations and optimal subject to a certain level of conservatism. Following the notation in Bertsimas and Sim (2004), we define a parameter $\Gamma_{t} \in \mathbb{R}^{+}$and a subset $S_{t}$ to control the level of conservatism in $W_{t}$, where $\Gamma_{t} \in\left[0,\left|J_{t}\right|\right], S_{t} \subseteq J_{t},\left|S_{t}\right|=\left\lfloor\Gamma_{t}\right\rfloor$, and $J_{t}=\{(i, j) \mid i=1, \ldots, n, j=$ $1, \ldots, t\}$. The rule of deviation for uncertain returns is defined, where one uncertain return's deviation can change up to $\left(\Gamma_{t}-\left\lfloor\Gamma_{t}\right\rfloor\right) \hat{g}_{v}(d, t),(v, d) \in J_{t} \backslash S_{t}$, and $\left\lfloor\Gamma_{t}\right\rfloor$ uncertain returns' deviations can change up to $\hat{g}_{i}(j, t),(i, j) \in S_{t}$. Intuitively, the robust optimization framework stipulates that only a subset of the uncertain coefficients will change and provides flexibility by adjusting the level of conservatism of the robust solution through the parameter $\Gamma_{t}$.

Given $\Gamma_{t}, S_{t}$ and $(v, d)$, the portfolio wealth $W_{t}$ at time $t, t=1, \ldots, T$, can be defined as

$$
W_{t}=\sum_{i=0}^{n} \sum_{j=1}^{t} \bar{g}_{i}(j, t) \xi_{i, j-1}-\left[\sum_{(i, j) \in S_{t}} \hat{g}_{i}(j, t)\left|\xi_{i, j-1}\right|+\left(\Gamma_{t}-\left\lfloor\Gamma_{t}\right\rfloor\right) \hat{g}_{v}(d, t)\left|\xi_{v, d-1}\right|\right]
$$

In order to make the solution to be robust against all scenarios of $\left\{S_{t} \cup\{(v, d)\}\left|S_{t} \subseteq J_{t},\right| S_{t} \mid=\right.$ $\left.\left\lfloor\Gamma_{t}\right\rfloor,(v, d) \in J_{t} \backslash S_{t}\right\}$, we develop the portfolio wealth that maximizes the derivation, which implies that the uncertain returns in Eq. (4) are equal to the worst case values in the scenarios of $\left\{S_{t} \cup\{(v, d)\}\left|S_{t} \subseteq J_{t},\right| S_{t} \mid=\left\lfloor\Gamma_{t}\right\rfloor,(v, d) \in J_{t} \backslash S_{t}\right\}$. Following Eq. (4), the robust counterpart of $W_{t}$ is given as

\footnotetext{
${ }^{3}$ While we focus on symmetric random uncertainty sets to illustrate the methodology in this paper, the approach could be naturally extended to asymmetric random uncertain sets for capturing the downside and upside risk as discussed in Chen et al. (2011).
} 


$$
\begin{aligned}
& W_{t}^{R}=\sum_{i=0}^{n} \sum_{j=1}^{t} \bar{g}_{i}(j, t) \xi_{i, j-1}- \\
& \max _{\left\{S_{t} \cup\{(v, d)\}\left|S_{t} \subseteq J_{t},\right| S_{t} \mid=\left\lfloor\Gamma_{t}\right\rfloor,(v, d) \in J_{t} \backslash S_{t}\right\}}\left\{\sum_{(i, j) \in S_{t}} \hat{g}_{i}(j, t)\left|\xi_{i, j-1}\right|+\left(\Gamma_{t}-\left\lfloor\Gamma_{t}\right\rfloor\right) \hat{g}_{v}(d, t)\left|\xi_{v, d-1}\right|\right\}
\end{aligned}
$$

Eq. (5) shows that the conservatism of the solution is controlled by the parameter $\Gamma_{t}$. When $\Gamma_{t}=0$, all uncertain returns are equal to $\bar{g}_{i}(j, t), i=1, \ldots, n, j=1, \ldots, t$, then Eq. (5) is equivalent to the nominal problem. When $\Gamma_{t}=n \cdot t$, all uncertain returns realize the highest deviations, then Eq. (5) is equivalent to the worst-case problem.

Bertsimas ans Sim (2004) proved that

$$
\max _{\left\{S_{t} \cup\{(v, d)\}\left|S_{t} \subseteq J_{t},\right| S_{t} \mid=\left\lfloor\Gamma_{t}\right\rfloor,(v, d) \in J_{t} \backslash S_{t}\right\}}\left\{\sum_{(i, j) \in S_{t}} \hat{g}_{i}(j, t)\left|\xi_{i, j-1}\right|+\left(\Gamma_{t}-\left\lfloor\Gamma_{t}\right\rfloor\right) \hat{g}_{v}(d, t)\left|\xi_{v, d-1}\right|\right\}
$$

is equivalent to

$$
\left\{\begin{aligned}
\max & \sum_{i=1}^{n} \sum_{j=1}^{t} \hat{g}_{i}(j, t)\left|\xi_{i, j-1}\right| z_{i, j} \\
\text { s.t. } & \sum_{i=1}^{n} \sum_{j=1}^{t} z_{i, j} \leq \Gamma_{t}, 0 \leq z_{i, j} \leq 1, i=1, \ldots, n, j=1, \ldots, t
\end{aligned}\right.
$$

By model (6), the robust wealth for risky asset $a_{i}$ at time $t$ is $x_{i, t}^{R}=\sum_{j=1}^{t} \bar{g}_{i}(j, t) \xi_{i, j-1}-$ $\hat{g}_{i}(j, t) z_{i, j}^{*}\left|\xi_{i, j-1}\right|$, where $\mathbf{z}_{t}^{*}$ represents the optimal solution in model $(6), i=1, \ldots, n, t=1, \ldots, T$. Thus, the robust formulation of the adjusted wealth for risky asset $a_{i}$ at time $t$ is $x_{i, t}^{+R}=x_{i, t}^{R}+\xi_{i, t}$, $i=1, \ldots, n, t=1, \ldots, T-1$.

To control the value of $\Gamma_{t}$ intuitively, similar to Bertsimas and Sim (2004), we use the notation of the violated probability. Let $W_{t}^{\text {opt }}$ and $\boldsymbol{\xi}_{t}^{*}$ denote the optimal value and the optimal solution of $\max _{\boldsymbol{\xi}_{t} \in D} W_{t}^{R}$, where $D$ denotes constraints in the portfolio. The violated probability is given as

$$
\operatorname{Prob}\left\{\sum_{i=0}^{n} \sum_{j=1}^{t} g_{i}(j, t) \xi_{i, j-1}^{*}<W_{t}^{o p t}\right\} \leq \varepsilon
$$

where $\varepsilon$ denotes the most violated probability. 
Eq. (7) provides a guarantee performance, where the maximum probability of the uncertain wealth $\sum_{i=0}^{n} \sum_{j=1}^{t} g_{i}(j, t) \xi_{i, j-1}^{*}$ less than $W_{t}^{o p t}$ is $\varepsilon$. The guarantee performance is a central feature in behavioral portfolio model (e.g. Shefrin and Statman, 2000; Das and Statman, 2013; Jiang et al, 2013). Bertsimas and Sim (2004) proved that Eq. (7) is equivalent to

$$
\Gamma_{t} \geq 1+\Phi^{-1}(1-\varepsilon) \sqrt{n \cdot t}
$$

where $\Phi$ denotes the cumulative distribution of the standard Gaussian random variable.

Using Eq. (8), the investor could determine the value of $\Gamma_{t}$ by $\varepsilon$. The most violated probability $\varepsilon$ is an intuitive index for the investor, which is similar to the confidence level in the value at risk $(\mathrm{VaR})$ and the conditional value-at-risk (CVaR) models. The investor could adjust the value of $\varepsilon$ to reflect his/her safety requirement and therefore control the conservation of the solution.

\subsection{Dynamic Prospect Theory Value Function}

We employ the prospect theory value function to reflect investors' behavior factors in the multi-period setting. In the framework of prospect theory (Kahneman and Tversky, 1979), the value function has three key characteristics. (1) Reference dependence: people evaluate assets by comparison with a given reference value. (2) Loss aversion: people are more sensitive to losses than to gains. (3) Diminishing sensitivity: people tend to be risk-averse in the domain of gains, while risk-seeking in the domain of losses. The prospect theory value function introduced by Kahneman and Tversky (1979) is expressed by

$$
P V(W)= \begin{cases}(W-\hat{y})^{\alpha}, & W \geq \hat{y} \\ -\lambda(\hat{y}-W)^{\beta}, & W<\hat{y}\end{cases}
$$

where $P V$ denotes the prospect theory value (PT value) function, $W$ denotes the portfolio wealth; $\lambda$ denotes the loss aversion ratio; $\hat{y}$ denotes the given reference wealth; $\alpha$ and $\beta$ denote the curvature parameters for gains and losses respectively. 4

Barberis and Huang (2001) defined a benchmark wealth which could be considered as an

\footnotetext{
${ }^{4}$ Tversky and Kahneman (1992) experimentally determined the values of $\alpha=\beta=0.88, \lambda=2.25$, which are considered as appropriate for describing most decision makers' behavior and used to make optimal decision-makings (cf. Fan et al., 2013; Krohling and de Souza, 2012).
} 
investor's memory of the earlier portfolio wealth. Following this framework, Fortin and Hlouskova (2011) suggested the benchmark wealth as the portfolio wealth of last period. Furthermore, they proposed the loss aversion parameters that are dynamically updated. If the current portfolio wealth is larger than the benchmark wealth, the investor will feel that the portfolio has performed well, then his/her loss aversion ratio is equal to the pre-defined loss aversion ratio and the given reference wealth is decreased. On the contrary, if the current portfolio wealth is smaller than the benchmark wealth, the investor will experience losses, then his/her loss aversion ratio is increased and the given reference wealth is equal to the pre-defined target wealth. The dynamically updated loss aversion parameters are given by

$$
\lambda_{t}=\left\{\begin{array}{ll}
\lambda^{0}, & W_{t}^{R} \geq W_{t-1}^{R} \\
\lambda^{0}+\left(\frac{W_{t-1}^{R}}{\left.W_{t}^{R}-1\right),}\right. & W_{t}^{R}<W_{t-1}^{R}
\end{array}, t=1, \ldots, T\right.
$$

and

$$
\hat{y}_{t}=\left\{\begin{array}{ll}
\frac{W_{t-1}^{R} W_{t}^{0},}{W_{t}^{R}} & W_{t}^{R} \geq W_{t-1}^{R} \\
W_{t}^{0}, & W_{t}^{R}<W_{t-1}^{R}
\end{array}, t=1, \ldots, T\right.
$$

where $\lambda_{t}$ denotes the loss aversion ratio at time $t, \hat{y}_{t}$ denotes the given reference wealth at time $t, W_{t}^{0}$ denotes the pre-defined target wealth at time $t, \lambda^{0}=2.25$ (Tversky and Kahneman, 1992; Fan et al., 2013; Krohling and de Souza, 2012).

In a multi-period investment problem, an investor usually determines a wealth target over the whole investment duration at the beginning of the investment (cf. Blake et al., 2013). Let $\bar{r}_{t}^{0}$ denote the target return of period $t$, thus the pre-defined target wealth at time $t$ can be obtained as $W_{t}^{0}=W_{0} \prod_{k=1}^{t}\left(1+\bar{r}_{k}^{0}\right), t=1, \ldots, T$. By Eqs. (9)-11), the dynamic prospect theory value function is defined as

$$
P V\left(W_{t}^{R}\right)=\left\{\begin{array}{ll}
\left(W_{t}^{R}-\hat{y}_{t}\right)^{\alpha}, & W_{t}^{R} \geq \hat{y}_{t} \\
-\lambda_{t}\left(\hat{y}_{t}-W_{t}^{R}\right)^{\beta}, & W_{t}^{R}<\hat{y}_{t}
\end{array}, t=1, \ldots, T\right.
$$


where $\lambda_{t}=\left\{\begin{array}{ll}\lambda_{t}^{0}, & W_{t}^{R} \geq W_{t-1}^{R} \\ \lambda_{t}^{0}+\left(\frac{W_{t-1}^{R}}{\left.W_{t}^{R}-1\right),}\right. & W_{t}^{R}<W_{t-1}^{R}\end{array}\right.$ and $\hat{y}_{t}= \begin{cases}\frac{W_{t-1}^{R}}{W_{t}^{R}} W_{t}^{0}, & W_{t}^{R} \geq W_{t-1}^{R} \\ W_{t}^{0}, & W_{t}^{R}<W_{t-1}^{R}\end{cases}$

\subsection{Formulation of the model}

Generally speaking, an investor's investment goal is to manage a portfolio in the manner that maximizes the PT value of the portfolio. More specifically, we quantify the total PT value as a weighted sum of PT value in each period. The objective function is expressed by

$$
\max \sum_{t=1}^{T} \omega_{t} \times P V\left(W_{t}^{R}\right)
$$

where $\omega_{t}$ is the target weight at period $t, \omega_{t} \geq 0, t=1, \ldots, T$.

Let $c_{i, t}$ denote the linear transaction cost for risky asset $a_{i}$ at time $t, i=1, \ldots, n, t=0, \ldots, T-$

1 , and the linear transaction cost for riskless asset $a_{0}$ is 0 , i.e. $c_{0, t}=0, t=0, \ldots, T-1.5$ Without loss of generality, suppose that the whole investment process is self-financing, that is, an investor does not invest additional capital or withdraw the old one during the investment duration. Based on the discussion above, an investment constraint is given by

$$
\sum_{i=0}^{n} \Delta x_{i, t}+\sum_{i=1}^{n} c_{i, t}\left|\Delta x_{i, t}\right|=0, t=0, \ldots, T-1
$$

Since the position invested in asset $i$ at time $t$ is an uncertain variable, $i=0,1, \ldots, n, t=$ $1, \ldots, T-1$, we use the robust counterpart to limit the position in each asset, which is known as the portfolio relative diversity constraints (cf. Calafiore, 2008). The constraints are given as

$$
l_{i} \leq \frac{x_{i, t}^{+R}}{W_{t}^{R}} \leq u_{i}, i=0,1, \ldots, n, t=0, \ldots, T-1
$$

where $x_{0, t}^{+R}=x_{0, t}^{+}, x_{i, 0}^{+R}=x_{i, 0}^{+}, W_{0}^{R}=W_{0}, u_{i}$ and $l_{i}$ denote the upper and lower bounds on the position of asset $a_{i}$ in the portfolio model, respectively.

\footnotetext{
${ }^{5}$ Generally, the transaction costs of bonds are included in the par value (The difference between the price a broker-dealer pays for a bond and the price at which it is sold to the investors is known as the bond's markup, which is the transaction cost. With new issues, the broker-dealer's markup is included in the par value), therefore investors do not need to pay separate transaction costs. Following Liu (2004) and Bertsimas and Pachamanova (2008), we assume the transaction cost for riskless asset $a_{0}$ is 0 .
} 
Following Eqs. (13) - (15), the robust multi-period portfolio model based on prospect theory is formulated as

$$
\begin{aligned}
\max & \sum_{t=1}^{T} \omega_{t} \times P V\left(W_{t}^{R}\right) \\
\text { s.t. } & \sum_{i=0}^{n} \Delta x_{i, t}+\sum_{i=1}^{n} c_{i, t}\left|\Delta x_{i, t}\right|=0, t=0, \ldots, T-1 \\
& l_{i} \leq \frac{x_{i, t}^{+R}}{W_{t}^{R}} \leq u_{i}, i=0,1, \ldots, n, t=0, \ldots, T-1
\end{aligned}
$$

\section{ALMV-PSO Algorithm}

Notice that model (16) is a complex nonlinear programming problem. As a result, the traditional robust optimization techniques (e.g. Ben-Tal and Nemirovski, 1998, 1999; Bertsimas and Sim, 2004) may fail to obtain the optimal solution. In order to solve the portfolio model effectively, we develop an ALMV-PSO algorithm.

Particle swarm optimization (PSO) is a population-based stochastic optimization method first proposed by Kennedy and Eberhart (1995) to solve nonlinear optimization problems. PSO has some computational advantages such as high efficiency in searching solutions and simpleness to implement. However, like other population-based algorithms, PSO is easy to be trapped into a local minima. The main reason for this problem is that the PSO algorithm is likely to lose the diversity during the searching process. Recently, Chen et al. (2013) proposed a PSO algorithm with an aging leader and Challengers (ALC-PSO), which introduced an aging leader to increase the diversity. Following observations from the nature that the mutation operator can also increase the diversity of a swarm, Pehlivanoglu (2013) designed a new PSO algorithm called multi-frequency vibrational PSO, which contains the multi-frequency vibrational mutation operator.

To enhance the diversity of the swarm in PSO, the ALMV-PSO algorithm considers both the aging leader and the multi-frequency vibrational mutation operator. In particular, since the initial positions based on the random initialization method (cf. Haddar et al., 2015; García-Hernández et al., 2015; Liu and Zhang, 2015) are likely to locate in an infeasible regime of the multi-period portfolio model, to initialize positions of a swarm in a feasible region and with a high level of 
diversity, we design a novel two-stage initialization strategy. Furthermore, the proposed portfolio model is a constrained optimization problem. For the sake of balancing the objective function value and the constraint violation function value in PSO algorithm, we also design an improved stochastic ranking approach, where the stochastic ranking approach proposed by Runarsson and Yao (2000) is not suitable for the searching rule of PSO algorithm. Next, we introduce the main operators in ALMV-PSO and the procedure of ALMV-PSO.

\subsection{Encoding and Initialization}

Let $\Delta x_{i, t}$, the adjustment wealth of risky asset $a_{i}$ at time $t$, be the decision variable, $i=1, \ldots, n$, $t=0, \ldots, T-1$. By Eq. 16b), the adjustment wealth of riskless asset $a_{0}$ at time $t$ is expressed by

$$
\Delta x_{0, t}=-\left(\sum_{i=1}^{n} \Delta x_{i, t}+\sum_{i=1}^{n} c_{i, t}\left|\Delta x_{i, t}\right|\right), t=0, \ldots, T-1
$$

A solution $\left[\Delta x_{1,0}, \ldots, \Delta x_{n, 0} ; \ldots ; \Delta x_{1, T-1}, \ldots, \Delta x_{n, T-1}\right]$ of model $[16]$ is encoded as a particle's position by a real-valued representation $\left[p_{1,0}^{0}, \ldots, p_{n, 0}^{0} ; \ldots ; p_{1, T-1}^{0}, \ldots, p_{n, T-1}^{0}\right]$. Let $\mathbf{P}^{k}(s)=$ $\left[p_{1,0}^{k}(s), \ldots, p_{n, 0}^{k}(s) ; \ldots ; p_{1, T-1}^{k}(s), \ldots, p_{n, T-1}^{k}(s)\right]$ and $\mathbf{V}^{k}(s)=\left[v_{1,0}^{k}(s), \ldots, v_{n, 0}^{k}(s) ; \ldots ; v_{1, T-1}^{k}(s), \ldots\right.$, $\left.v_{n, T-1}^{k}(s)\right]$ denote the position and the velocity of particle $s$ at iteration $k$, respectively.

In order to make initial positions of a swarm in a feasible region and with a high level of diversity, we propose a two-stage initialization strategy, including the multi-period initialization stage and the diversification stage.

(1) Multi-period initialization stage

The robust formulation of the adjusted wealth for risky asset $a_{i}$ at time $t$ is expressed by $x_{i, t}^{+R}=\sum_{j=1}^{t} \bar{g}_{i}(j, t) \xi_{i, j-1}-\hat{g}_{i}(j, t) z_{i, j}^{*}\left|\xi_{i, j-1}\right|+\xi_{i, t}$, where $\xi_{i, 0}=x_{i, 0}+\Delta x_{i, 0}, \xi_{i, t}=\Delta x_{i, t}, \mathbf{z}_{t}^{*}$ represents the optimal solution in model (6), $i=1, \ldots, n, t=1, \ldots, T-1$. It shows that whether $x_{i, t}^{+R}$ satisfies Eq. (16c), it depends on the adjustment wealth of both the current period $\Delta x_{i, t}$ and the prior period $\Delta x_{i, j}, j=0, \ldots, t-1$. Thus, to guarantee that all initial positions are in the feasible region of model (16), the current period position $\left[p_{1, t}^{0}, \ldots, p_{n, t}^{0}\right]$ needs to be initialized based on prior period positions $\left[p_{1,0}^{0}, \ldots, p_{n, 0}^{0} ; \ldots ; p_{1, t-1}^{0}, \ldots, p_{n, t-1}^{0}\right]$. Following the discussion above, the multi-period initialization stage is designed as follows: 6

\footnotetext{
${ }^{6}$ For notational convenience, let $x_{0, t}^{R}=x_{0, t}, x_{i, 0}^{R}=x_{i, 0}, t=0, \ldots, T-1, i=0, \ldots, n$.
} 
Step 1. Let $s=1$.

Step 2. Let $t=0$, and denote the portfolio at time $t$ as $\left[x_{0, t}^{R}, x_{1, t}^{R}, \ldots, x_{n, t}^{R}\right]$.

Step 3. Randomly generate an initial position $\left[p_{1, t}^{0}(s), \ldots, p_{n, t}^{0}(s)\right]$ at time $t$ based on portfolio $\left[x_{0, t}^{R}, x_{1, t}^{R}, \ldots, x_{n, t}^{R}\right]$, where $\left[p_{1, t}^{0}(s), \ldots, p_{n, t}^{0}(s)\right]$ satisfies Eq. (16c). Furthermore, calculate the portfolio $\left[x_{0, t+1}^{R}, x_{1, t+1}^{R}, \ldots, x_{n, t+1}^{R}\right]$ based on the position $\left[p_{1,0}^{0}(s), \ldots, p_{n, 0}^{0}(s) ; \ldots ; p_{1, t}^{0}(s), \ldots, p_{n, t}^{0}(s)\right]$.

Step 4. Let $t=t+1$. If $t \leq T-1$, then return to Step 3; otherwise, we obtain the initial position of particle $s$ (denoted by $\mathbf{P}^{0}(s)$ ), and go to Step 5 .

Step 5. Let $s=s+1$. If $s \leq S_{m}$, then return to Step 2, where $S_{m}$ denotes the number of position generated in the multi-period initialization stage; otherwise, terminate and obtain the $S_{m}$ initial positions denoted by $Z_{S_{m}}^{0}=\left\{\mathbf{P}^{0}(1), \ldots, \mathbf{P}^{0}\left(S_{m}\right)\right\}$.

(2) Diversification stage

After the multi-period initialization stage, all initial positions are in the feasible region of model (16). It is well known that positions with a high level of diversity would enhance the performance of PSO (cf. Pehlivanoglu, 2013; Chen et al., 2013). In order to enhance the diversity of positions, we choose the $S$ most diversified positions from $Z_{S_{m}}^{0}$, where $S$ denotes the swarm size in the ALMV-PSO algorithm. Following the approach of Hassanzadeh et al. (2014), the diversification stage is described as follows:

Step 1. Calculate $\pi_{i, t}=\frac{\left(\bar{p}_{i, t}-\underline{p}_{i, t}\right)^{-1}}{\sum_{t=0}^{T-1} \sum_{i=1}^{n}\left(\bar{p}_{i, t}-\underline{p}_{i, t}\right)^{-1}}$, where $\bar{p}_{i, t}=\max \left\{p_{i, t}^{0}(s) \mid s=1, \ldots, S_{m}\right\}$, $\underline{p}_{i, t}=\min \left\{p_{i, t}^{0}(s) \mid s=1, \ldots, S_{m}\right\}$. Randomly select a position from $Z_{S_{m}}^{0}$, and transfer it into the set $Z_{S}^{0}$, which is used to save the most diversified positions that have been selected.

Step 2. Find $\mathbf{P}^{\max } \in Z_{S_{m}}^{0}$, where $\mathbf{P}^{\max }=\left[p_{1,0}^{\max }, \ldots, p_{n, 0}^{\max } ; \ldots ; p_{1, T-1}^{\max }, \ldots, p_{n, T-1}^{\max }\right]$, so that $\mathbf{P}^{\max }$ is the most diversified position from all positions in $Z_{S}^{0}$, that is

$$
\left[\sum_{t=0}^{T-1} \sum_{i=1}^{n}\left(\pi_{i, t}\left|p_{i, t}^{\max }-p_{i, t}^{d}\right|\right)^{2}\right]^{\frac{1}{2}}=\max _{\mathbf{P} \in Z_{S_{m}}^{0}} \min _{\mathbf{P}^{d} \in Z_{S}^{0}}\left[\sum_{t=0}^{T-1} \sum_{i=1}^{n}\left(\pi_{i, t}\left|p_{i, t}-p_{i, t}^{d}\right|\right)^{2}\right]^{\frac{1}{2}}
$$

where $\mathbf{P}=\left[p_{1,0}, \ldots, p_{n, 0} ; \ldots ; p_{1, T-1}, \ldots, p_{n, T-1}\right], \mathbf{P}^{d}=\left[p_{1,0}^{d}, \ldots, p_{n, 0}^{d} ; \ldots ; p_{1, T-1}^{d}, \ldots, p_{n, T-1}^{d}\right]$.

Step 3. Transfer $\mathbf{P}^{\max }$ to $Z_{S}^{0}$. If $\left|Z_{S}^{0}\right|=S$, then terminate and obtain the $S$ diversified positions denoted by $Z_{S}^{0}=\left\{\mathbf{P}^{0}(1), \ldots, \mathbf{P}^{0}(S)\right\}$; otherwise, return to Step 2 .

The initial positions in $Z_{S}^{0}$ satisfy the constraints in model (16), and have a high level of diversity. We randomly generate the initial velocity of particle $s$ denoted by $\mathbf{V}^{0}(s)=\left[v_{1,0}^{0}(s), \ldots, v_{n, 0}^{0}(s)\right.$; 
$\left.\ldots ; v_{1, T-1}^{0}(s), \ldots, v_{n, T-1}^{0}(s)\right], s=1, \ldots, S$, where $-V \max \leq v_{i, t}^{0}(s) \leq V \max$, and $V \max$ denotes the maximum value of the velocity.

\subsection{Stochastic Ranking}

Model (16) is a constrained optimization problem, which needs to handle the constraints in the searching process. The objective function $f$ and constraint violation function $\ell$ are given by

$$
\begin{gathered}
f\left(\mathbf{P}^{k}(s)\right)=\sum_{t=1}^{T} \omega_{t} \times P V\left(W_{t}^{R}\right) \\
\ell\left(\mathbf{P}^{k}(s)\right)=\sum_{t=1}^{T} \sum_{i=0}^{n} \max \left\{U_{i, t}\left(\mathbf{P}^{k}(s)\right), 0\right\}+\sum_{t=1}^{T} \sum_{i=0}^{n} \max \left\{L_{i, t}\left(\mathbf{P}^{k}(s)\right), 0\right\}
\end{gathered}
$$

where $U_{i, t}(\mathbf{P})=\frac{x_{i, t}^{+R}}{W_{t}^{R}}-u_{i}, L_{i, t}(\mathbf{P})=l_{i}-\frac{x_{i, t}^{+R}}{W_{t}^{R}}, k=0, \ldots, K_{\max }, K_{\max }$ is the maximum iteration number.

The penalty function method is the most common method used in constrained optimization problems, and penalties individuals based on their constraint violation function value (cf. Liu et al., 2012; Venkatraman and Yen, 2005). In the penalty function method, however, it is very difficult to find the optimal penalty coefficients for balancing between the objective function value and the constraint violation function value. To overcome this difficulty, Runarsson and Yao (2000) proposed a stochastic ranking approach, which introduces a probability factor $P_{f}$ of using only the objective function for comparisons in ranking the infeasible regions of the search space. That is, given any pair of two adjacent individuals, the probability of comparing them according to the objective function is 1 if both individuals are feasible, otherwise is $P_{f}$. The pseudo-code of stochastic ranking approach proposed by Runarsson and Yao (2000) is illustrated in Fig. 2.

[Insert Figure 2 Here]

The stochastic ranking approach for Runarsson and Yao (2000) is suitable for $(\mu, \lambda)$-evolutionary algorithm, which ranks the individuals based on current position and selects the best $\mu$ individuals out of $\lambda$ individuals for the next generation. However, ALMV-PSO algorithm needs to rank 
the particles based on the historical positions and only selects the best individual. Inspired by Runarsson and Yao (2000), we design an improved stochastic ranking approach for PSO algorithm.

In the improved stochastic ranking approach, we introduce two probability factors, which are $P_{i n f}$ and $P_{o b j}$. The probability factor $P_{i n f}$ stands for the probability of choosing the infeasible individuals, while the probability factor $P_{o b j}$ stands for the probability of using the objective function for comparing the infeasible individuals. That is, for the infeasible individuals, the probability of comparing them according to the objective function is $P_{o b j}$. Next, the algorithm could choose an infeasible individual with the probability $P_{\text {inf }}$. The pseudo-code of the improved stochastic ranking approach for updating the personal best solution (denoted by pbest) and the global best solution (denoted by gbest) are illustrated in Fig. 3 and Fig. 4 , respectively.

$$
\text { [Insert Figure 3 \& Figure 4 Here] }
$$

Different from basic PSO algorithm, there is a leader of swarm (denoted by Leader) in ALMVPSO. Leader represents the best solution generated by particles during the leader's lifetime, while gbest represents the best solution generated by particles during the overall searching process. Therefore, the update procedure for Leader is similar to the update procedure for gbest.

\subsection{Velocity and Position Update}

In the ALC-PSO algorithm (Chen et al., 2013), each particle's velocity and position are updated through the pbest and the Leader. Following the ALC-PSO, the update rule of velocity and position in the ALMV-PSO are given by

$$
\begin{gathered}
v_{i, t}^{k+1}(s)=w v_{i, t}^{k}(s)+c_{1} r_{1}\left(\text { pbest }_{i, t}^{k}(s)-p_{i, t}^{k}(s)\right)+c_{2} r_{2}\left(\text { Leader }_{i, t}^{k}-p_{i, t}^{k}(s)\right) \\
p_{i, t}^{k+1}(s)=p_{i, t}^{k}(s)+v_{i, t}^{k+1}(s)
\end{gathered}
$$

where $w$ is the inertia weight, both $c_{1}$ and $c_{2}$ are the cognitive coefficients, both $r_{1}$ and $r_{2}$ are random numbers uniformly distributed in the interval $[0,1]$. 


\subsection{Global and Local Mutation}

Pehlivanoglu (2013) introduced a multi-frequency vibrational mutation operator, which is used to conduct the global and local mutation in PSO. While the multi-frequency vibrational mutation method (Pehlivanoglu, 2013) increases the diversity of swarm, it is not developed for a multi-period portfolio model, in which case all particles' positions are likely to be out of the feasible region by using the global mutation. We develop an alternative global mutation, which is expressed by

$$
\left\{\begin{array}{l}
p_{i, t}^{k}(s)=p_{i, t}^{k}(s)+V \max \times\left[1+A_{1}(0.5-\operatorname{rand} n) \delta\right] \\
\delta= \begin{cases}1, & k=\lambda \times f r_{1}, \lambda=1,2, \ldots \\
0, & k \neq \lambda \times f r_{1}, \lambda=1,2, \ldots\end{cases}
\end{array}\right.
$$

where $A_{1}$ is the amplitude factor of global mutation, randn is a random number distributed in accordance with $N[0,1]$, and $f r_{1}$ is the frequency of global mutation.

In Eq. (23), each particle moves in the range $\left[\operatorname{Vmax}\left(1-0.5 A_{1}\right), \operatorname{Vmax}\left(1+0.5 A_{1}\right)\right]$, which makes the particle tend to search in the feasible region. The local mutation proposed by Pehlivanoglu (2013) is expressed as

$$
\left\{\begin{array}{l}
p_{i, t}^{k}(q)=\text { gbest }_{i, t}\left[1+A_{2}(0.5-\operatorname{randn}) \delta\right] \\
\delta= \begin{cases}1, & k=\lambda \times f r_{1}, \lambda=1,2, \ldots \\
0, & k \neq \lambda \times f r_{1}, \lambda=1,2, \ldots\end{cases}
\end{array}\right.
$$

where $q=1, \ldots, Q, Q$ is the amount of particles executing local mutation, $A_{2}$ is the amplitude factor of local mutation, $f r_{2}$ is the frequency of local mutation.

Pehlivanoglu (2013) shown PSO has better performance to control the local mutation, which may improve the quality of the particle generated by Eq. (24). We generate $Q$ particles through Eq. 24) and rank the $Q$ particles by the stochastic ranking approach (Runarsson and Yao, 2000). The $Q_{s}$ particles, which have the best rank in $Q$ particles, are randomly placed into the new swarm.

\subsection{Lifespan Control and New Leader Generation}

Based on the leader's leading power, the ALC-PSO algorithm (Chen et al., 2013) uses an operator of lifespan control to adjust the leader's lifespan. Let $\Theta$ denote the lifespan of the leader, 
and $\Theta_{0}$ denote the initial value of $\Theta$. We determine the leader's leading power by the improved ratio, which is expressed by

$$
\operatorname{Iratio}(k)=\frac{f(\text { best_feasible }(k))-f\left(\text { best_feasible }_{-}(k-1)\right)}{\mid f\left(\text { best_feasible }_{-}(k-1)\right) \mid}
$$

where $\operatorname{Iratio}(k)$ denotes the improved ratio at iteration $k$, best_feasible $(k)$ denotes the feasible particle with the best objective function value during $k$ iterations.

Based on the significance of the leader in terms of its searching performance, scenarios of improvement are categorized into the following four cases. Case 1: the leader has a good leading power $\left(\operatorname{Iratio}(k)>10^{-4}\right)$. Case 2 : the leader has a fair leading power $\left(10^{-6}<\operatorname{Iratio}(k) \leq 10^{-4}\right)$. Case 3: the leader has a poor leading power $\left(0<\operatorname{Iratio}(k) \leq 10^{-6}\right)$. Case 4 : the leader has no leading power $(\operatorname{Iratio}(k)=0)$. Following the method of Chen et al. (2013), the ALMV-PSO algorithm adjusts the lifespan based on the above four cases, as described in Fig. 5.

\section{[Insert Figure 5 Here]}

Let $\theta$ be the age of the leader, and the initial age $\theta=0$. When the iteration number increases by 1 , the leader's age also increases by 1 . If $\theta=\Theta$, then the algorithm needs to generate a new leader. Different from Chen et al. (2013), we randomly choose a particle from the current swarm as the new leader. In our approach, the operator of the new leader generation is easy to implement and makes the learning object to be diversified for the ALMV-PSO algorithm.

\subsection{Procedure of ALMV-PSO}

Summarizing the aforementioned operators, there are six steps in the ALMV-PSO algorithm:

Step 1. Determine parameters: $S_{m}, S, V \max , K_{\max }, \xi_{\max } c_{1}, c_{2}, w, P_{f}, P_{\text {inf }}, P_{\text {obj }}, f r_{1}, f r_{2}$, $A_{1}, A_{2}, \Theta_{0}, Q$ and $Q_{s}$. Set iteration number $k=0$, and successive poor iteration number $\xi=0.7$

Step 2. Initial $S$ particles' positions $\left\{\mathbf{P}^{0}(1), \ldots, \mathbf{P}^{0}(S)\right\}$, which follow the two-stage initialization strategy. Update the pbest, gbest and Leader by the improved stochastic ranking approach. Set the leader's age $\theta=0$, and the lifespan $\Theta=\Theta_{0}$.

\footnotetext{
${ }^{7}$ While the improved ratio is less than $10^{-6}$, we consider that the current iteration has a poor performance. $\xi_{\max }$ denotes the maximum number of the successive poor iteration.
} 
Step 3. If $k=f r_{1}$, then execute the operator of the global mutation using Eq. (23) and go to Step 5; otherwise, go to Step 4.

Step 4. Update the swarm's velocities and positions using Eq. (21) and Eq. (22) respectively. When $k=f r_{2}$, execute the operator of the local mutation using Eq. (24).

Step 5. Update the pbest, gbest and Leader by the improved stochastic ranking approach. Execute the operator of the lifespan control. Set $\theta=\theta+1$. when $\theta=\Theta$, execute the operator of the new leader generation, set the new leader's age $\theta=0$ and the lifespan $\Theta=\Theta_{0}$.

Step 6. If $\operatorname{Iratio}(k) \geq 10^{-6}$, then set $\xi=0$; otherwise, set $\xi=\xi+1$. Set $k=k+1$. If $k \leq K_{\max }$ and $\xi \leq \xi_{\max }$, then return to Step 3; otherwise, terminate and output best_feasible $(k) !^{8}$

\section{Numerical Illustration}

In this section, we illustrate the feasibility of the proposed model in a multi-period problem and check the effectiveness of the ALMV-PSO algorithm using real market data. Assume that an investor wants to construct a four-period investment strategy $(T=4)$, and the length of each period is 1-quarter. The investment duration is from January 2014 to December 2014. The investor initially holds $\$ 1,000,000$ wealth of the riskless asset.

The data set used in this study consists of 136 quarterly returns of all 32 stocks listed in the current S\&P 100 that have complete data during the period of January 1981 to December 2014, where the data before January 2014 is chosen as the in-sample data. The 3-month U.S. Treasury bill is used as a riskless asset, thus $\bar{r}_{0, t}=0.0175 \%, t=1,2,3,4.9$ Suppose that the transaction cost of risky asset $c_{i, t}$ is $1 \%, i=1, \ldots, 32$, the target weights $\omega_{t}=1$, the investor's target return at each period $\bar{r}_{t}^{0}$ is $1 \%, t=1,2,3,4$, and the upper bound $u_{i}$ and the lower bound $l_{i}$ on the weight of asset $a_{i}$ are 0.3 and 0 respectively, $i=0,1, \ldots, 32$. Stock price data is available from the Center for Research in Securities Prices (CRSP).

The parameter settings for ALMV-PSO are described as follows: $S_{m}=300, \operatorname{Vmax}=1,000$, $K_{\max }=10,000, \xi_{\max }=1,000, c_{1}=c_{2}=2, w=0.4, P_{f}=0.45, P_{\text {inf }}=0.45, P_{o b j}=0.5, f r_{1}=50$,

\footnotetext{
${ }^{8}$ If $\xi>\xi_{\max }$, we consider that the algorithm has already converged.

${ }^{9}$ The annualized return of 3-month U.S. Treasury bill is $0.07 \%$ on Dec 31,2013 , thus the quarterly return is $0.0175 \%$.
} 
$f r_{2}=20, A_{1}=A_{2}=0.5, \Theta_{0}=50, Q=30, Q_{s}=10 .{ }^{10}$

\subsection{Evaluation of Stock Returns}

Following the definition in Bertsimas and Sim (2004) and Gregory et al. (2011), we model the uncertain return as a random variable that has an arbitrary symmetric distribution in the interval $\left[\bar{g}_{i, t}-2 \sigma_{i, t}, \bar{g}_{i, t}+2 \sigma_{i, t}\right]$, where $\bar{g}_{i, t}$ denotes the mean cumulative return of risky asset $a_{i}$, and $\sigma_{i, t}$ denotes the standard deviation of the cumulative return of risky asset $a_{i}, i=1, \ldots, 32$, $t=1,2,3,4$. The sample period is from January 1981 to December 2013.

\subsection{Computational Results and Comparison of Algorithms}

To evaluate more precisely the impact of each proposed new component on the ALMV-PSO algorithm, we present three variants of the PSO algorithm proposed by Kennedy (1997) (PSO-1, PSO-2, and PSO-3) for comparisons. In the PSO-1 algorithm, the random initialization method (cf. Haddar et al., 2015; García-Hernández et al., 2015; Liu and Zhang, 2015) is applied to initialize the positions of the swarm, and the improved stochastic ranking approach is used. In the PSO-2 algorithm, the penalty method in Liu et al. (2012) is applied to measure the fitness of the swarm, and the two-stage initialization strategy is used. In the PSO-3 algorithm, both of the two-stage initialization strategy and the improved stochastic ranking approach are used.

The PSO algorithms are employed to solve model (16) under the value of $\varepsilon$ from 0.05 to 0.20 , and all algorithms are run 10 times independently. ${ }^{11}$ The average objective value, the best objective value, and the mean CPU running time are reported as indicators for comparing the performance of different algorithms. The results are shown in Table 1.

\section{$\underline{\text { [Insert Table } 1 \text { Here] }}$}

(1) Comparison of PSO-1 and PSO-3

Table 1 shows that the average objective value and the best objective value of PSO-3 are both better than PSO-1. Since whether $x_{i, t}^{+R}$ satisfies Eq. 16c depends on the adjustment wealth of

\footnotetext{
${ }^{10}$ All experiments in this paper are conducted in Matlab R2010a on a PC with Intel Core 2 CPU and 4G RAM.

${ }^{11}$ The value of the most violated probability is determined by investors' risk preference and the goal of the investment. The value of $\varepsilon$ means that the performance of the portfolio can be achieved up to the probability value of $1-\varepsilon$ (Bertsimas and Thiele, 2006). By the value of $\varepsilon$, the value of $\Gamma_{t}$ can be determined indirectly using Eq. (8).
} 
both the current period $\Delta x_{i, t}$ and the prior period $\Delta x_{i, j}, j=0, \ldots, t-1$, it is likely that the initial positions based on the random initialization method are infeasible. The two-stage initialization strategy enables the investors to make initial positions of a swarm into a feasible region and with a high level of diversity. By comparing the results of PSO-1 with PSO-3, it shows that the PSO algorithm combines with the two-stage initialization strategy is more suitable than the random initialization method to addressing the multi-period portfolio problems.

(2) Comparison of PSO-2 and PSO-3

By comparing the results between PSO-2 and PSO-3 in Table 1, it shows that both of the average objective value and the best objective value of PSO-3 outperform PSO-2 significantly. Different from the penalty method which requires a difficult optimization problem to find the optimal penalty coefficients (Runarsson and Yao, 2000), the improved stochastic ranking approach automatically balances between the objective function value and the constraint violation function value for the constrained portfolio problem. Thus, the improved stochastic ranking approach is more suitable for handling the constrained optimization problems.

(3) Comparison of PSO-3 and ALMV-PSO

From Table 1 above, although the mean CPU time of ALMV-PSO is more than PSO-3, both of the average objective value and the best objective value of ALMV-PSO are better than PSO-3. This is in agreement with the no-free-lunch theorem proposed by Wolpert and Macready (1997). By comparing the performance of ALMV-PSO with PSO-3, it shows that the aging leader and the multi-frequency vibrational mutation operator can further enhance the performance of PSO. Therefore, ALMV-PSO is effective to solve the robust multi-period portfolio model.

\subsection{Performance of Portfolio Model}

Under the real multi-period investment environment, Calafiore (2008) showed that only action $\Delta \mathbf{X}(0)$ is actually applied at $t=0$. At $t=1$, the actual outcome of asset returns over the first period is revealed to the investor, and therefore he/she will reconstruct another multi-period portfolio taking this knowledge into account. Following Calafiore (2008), the only action $\Delta \mathbf{X}(0)$ is taken at $t=0$, and then the wealth of assets $\mathbf{X}(1)$ is calculated based on the actual return at period 1. At $t=1, \mathbf{X}(1)$ turns into the initial portfolio and the new multi-period portfolio is constructed based on the proposed portfolio model. This process is repeated until the multi-period 
investment is finished $(t=4)$.

(1) Robustness analysis

One of the main goals for the robust optimization approach is to address the issue of investment performance, where the optimal performance of portfolio model is sensitive to the estimated parameters. To study the robustness of the proposed portfolio model, we adopt the robustness measure in Kim et al. (2014) defined by the performance fluctuation from the change in uncertain parameters. The portfolios that are robust will have small performance fluctuations and therefore relative low levels for the robustness measure.

In order to discuss the robustness measure of the proposed portfolio model, we compare it with the nominal portfolio model, where the nominal portfolio model is modeled by Eq. (16) with $\Gamma_{t}=0, t=1,2,3,4$. The robustness measures of the portfolio models are shown in Table 2 .

\section{[Insert Table 2 Here]}

By comparing the robust portfolios with the nominal portfolio, Table 2 shows that the robustness measure of the nominal portfolio is significantly larger than the robust portfolios, and confirms that the performance of the nominal portfolio is more sensitive to the estimated parameters. Therefore, the nominal portfolio is less preferable for investors in practice. Table 2 also shows that the portfolio with a larger level of conservation will have a lower level of robustness measure, which is consistent with the idea of the robust optimization method.

(2) Investment analysis

To further test the effectiveness of the robust multi-period portfolio model, under the real multi-period investment, the actual wealth and the robust wealth at each period are compared 12 The results are shown in Table 3 .

\section{[Insert Table 3 Here]}

From Table 3, we find that the actual wealth is larger than the robust wealth at each period. Therefore, investors can consider the robust wealth as the safety wealth in the real investment, which is an important feature in wealth management. Moreover, we also find that the ex-post

\footnotetext{
${ }^{12}$ The actual wealth is calculated by adding up the actual wealth of assets at each period. The robust wealth is represented by $W_{t}^{R}$ at Eq. (5).
} 
return of the proposed model in Table 3 outperforms the S\&P 100 index return of $12.38 \%$ in the investment horizon from January 2014 to December 2014.

Therefore, through the robust multi-period portfolio model based on prospect theory, an investor can develop a robust multi-period portfolio strategy that satisfies his/her behavioral characteristics and are effective in the real multi-period investment.

\section{Conclusion}

This paper develops a novel robust multi-period portfolio selection model, which considers investors' behavioral characteristics. By integrating the robust theory and prospect theory, a robust multi-period portfolio considering investors' behavioral factors is constructed, which not only addresses the parameter uncertainty, but also features the reference dependence, loss aversion and diminishing sensitivity. Firstly, considering the uncertainty of security returns, we employ the robust optimization approach of Bertsimas and Sim (2004) to formulate a robust multi-period portfolio, which introduces a parameter $\varepsilon$ to indirectly adjust the degree of conservatism of the robust solutions. Secondly, a dynamic prospect theory value function is proposed, where the loss aversion parameters are updated dynamically. Furthermore, based on prospect theory, a robust multi-period portfolio that considers investors' behavioral factors is constructed, which features the reference dependence, loss aversion and diminishing sensitivity. Thirdly, in order to solve the multiperiod portfolio model, we develop an ALMV-PSO algorithm. In the ALMV-PSO algorithm, an aging leader and the multi-frequency vibrational mutation operator are employed, which can reduce the probability of being trapped in local optima. In addition, a two-stage initialization strategy and an improved stochastic ranking approach for PSO are proposed. The two-stage initialization strategy guarantees that the initial positions are in the feasible region and with a high level of diversity. The improved stochastic ranking approach balances between the objective function value and the constraint violation function value. Finally, a real market data example is given to illustrate the feasibility of the proposed model and check the effectiveness of the ALMV-PSO algorithm in a multi-period problem. The results show that the ALMV-PSO algorithm is successful in solving the proposed model and the proposed model can develop a multi-period strategy, which satisfies investors' behavioral characteristics and are effective in the real multi-period investment. 
Compared with three variants of PSO algorithm, the proposed algorithm has three main advantages: 1) the two-stage initialization strategy is more suitable than the random initialization method in PSO algorithm to addressing the multi-period portfolio problems. 2) the improved stochastic ranking approach is more suitable for handling the constrained optimization problems comparing with the penalty method in the PSO algorithm. 3) the aging leader and multi-frequency vibrational mutation operator enhance the performance of the PSO algorithm. Additionally, the proposed robust portfolio is less sensitive to the estimated parameters in comparison with the nominal portfolio, and the ex-post return of the proposed model outperforms the S\&P 100 index in the investment horizon of the study.

The proposed robust multi-period portfolio model opens future research avenues for further improvements and incorporation of new constrains in reality that is not considered in the current model. For example, we have followed the literature to consider a single objective model. There might be times when a portfolio decision is desired to be with a multi-objective alternative that simultaneously considers the PT value, liquidation risk, social responsibility and so on. In addition, the current framework could be naturally extended to incorporate the minimum transaction lots, tax and cardinality constraints, which exist in real world. Moreover, regime-switching could be incorporated into the current framework so that the return distributions are dynamic with respect to different regimes. Finally, the proposed robust multi-period portfolio model can be extended in light of the new data-driven portfolio method (Calafiore 2013), where the assumption of assets' distributions is not necessary. These interesting future research topics can be built up on the same conceptual and technical grounds of our current modeling framework. 


\section{Acknowledgement}

We are appreciative for the financial support by National Science Foundation of China (No. 71372186, 71271047 and 70901017), Program for New Century Excellent Talents in University (NCET-13-0115), Program for Liaoning Excellent Talents in University (LJQ2013030) and Fundamental Research Funds for the Central Universities (N120506002, N130606002). 


\section{References}

[1] Ai, J., Brockett, P., \& Wang, T. (2013) Optimal Enterprise Risk Management and Decision Making with Shared and Dependent Risks. http://ssrn.com/abstract $=2352485$

[1] Babaei, S., Sepehri, M. M., \& Babaei, E. (2015). Multi-objective portfolio optimization considering the dependence structure of asset returns. European Journal of Operational Research, 244, 525-539.

[2] Barberis, N., \& Huang, M. (2001). Mental accounting, loss aversion, and individual stock returns. The Journal of Finance, 56, 1247-1292.

[3] Ben-Tal, A., \& Nemirovski, A. (1998). Robust convex optimization. Mathematics of Operations Research, 23, 769-805.

[4] Ben-Tal, A., \& Nemirovski, A. (1999). Robust solutions of uncertain linear programs. Operations Research Letters, 25, 1-13.

[5] Bernard, C., \& Vanduffel, S. (2014). Mean-variance optimal portfolios in the presence of a benchmark with applications to fraud detection. European Journal of Operational Research, 234, 469-480.

[6] Bertsimas, D., \& Sim, M. (2004). The price of robustness. Operations Research, 52, 35-53.

[7] Bertsimas, D., \& Thiele, A. (2006). Robust and data-driven optimization: Modern decisionmaking under uncertainty. INFORMS Tutorials in Operations Research, 95-122.

[8] Bertsimas, D., \& Pachamanova, D. (2008). Robust multiperiod portfolio management in the presence of transaction costs. Computers \& Operations Research, 35, 3-17.

[9] Best, M. J., \& Grauer, R. R. (1991). Sensitivity analysis for mean-variance portfolio problems. Management Science, 37, 980-989.

[10] Black, F., \& Litterman, R. (1992). Global portfolio optimization. Financial Analysts Journal, 48, 28-43.

[11] Blake, D., Wright, D., \& Zhang, Y. (2013). Target-driven investing: Optimal investment strategies in defined contribution pension plans under loss aversion. Journal of Economic Dynamics \& Control, 37, 195-209.

[12] Calafiore, G. C. (2008). Multi-period portfolio optimization with linear control policies. Automatica, 44, 2463-2473.

[13] Calafiore, G. C. (2013). Direct data-driven portfolio optimization with guaranteed shortfall probability. Automatica, 49, 370-380.

[14] Chen, C., \& Kwon, R. H. (2012). Robust portfolio selection for index tracking. Computers \& Operations Research, 39, 829-837.

[15] Chen, W., Tan, S., \& Yang, D. (2011). Worst-case VaR and robust portfolio optimization with interval random uncertainty set. Expert Systems with Applications, 38, 64-70.

[16] Chen, W. N., Zhang, J., Lin, Y., Chen, N., Zhan, Z. H., Chung, H. S. H., Li, Y., \& Shi, Y. 
H. (2013). Particle swarm optimization with an aging leader and challengers. IEEE Transactions on Evolutionary Computation, 17, 241-258.

[17] Das, S. R., \& Statman, M. (2013). Options and structured products in behavioral portfolios. Journal of Economic Dynamics and Control, 37, 137-153.

[18] Fan, Z. P., Zhang, X., Chen, F. D., \& Liu, Y. (2013). Multiple attribute decision making considering aspiration-levels: A method based on prospect theory. Computers \& Industrial Engineering, 65, 341-350.

[19] Fortin, I., \& Hlouskova, J. (2011). Optimal asset allocation under linear loss aversion. Journal of Banking \& Finance, 35, 2974-2990.

[20] Gabrel, V., Murat, C., \& Thiele, A. (2014). Recent advances in robust optimization: An overview. European Journal of Operational Research, 235, 471-483.

[21] García-Hernández, L., Palomo-Romero, J. M., Salas-Morera, L., Arauzo-Azofra, A. \& Pierreval, H. (2015). A novel hybird evolutionary approach for capturing decision maker knowledge into the unequal area facility layout problem. Expert Systems with Applications, 42, 4697-4708.

[22] Gharakhani, M., \& Sadjadi, S. (2011). A new approach to robust modeling of the multiperiod portfolio problem. African Journal of Business Management, 5, 9998-10006.

[23] Gregory, C., Darby-Dowman, K., \& Mitra, G. (2011). Robust optimization and portfolio selection: The cost of robustness. European Journal of Operational Research, 212, 417-428.

[24] Guo, W. (2014). Optimal portfolio choice for an insurer with loss aversion. Insurance: Mathematics and Economics, 58, 217-222.

[25] Haddar, B., Khemakhem, M., Hanafi, S., \& Wilbaut, C. (2015). A hybird heuristic for the 0-1 knapsack sharing problem. Expert systems with Applications, 42, 4653-4666.

[26] Hassanzadeh, F., Nemati, H., \& Sum, M. (2014). Robust optimization for interactive multiobjective programming with imprecise information applied to R\&D project portfolio selection. European Journal of Operational Research, 238, 41-53.

[27] Heiman, A., Just, D. R., McWilliams, B. P., \& Zilberman, D. (2015). A prospect theory approach to assessing changes in parameters of insurance contracts with an application to moneyback guarantees. Journal of Behavioral and Experimental Economics, 54, 105-117.

[28] Hoffmann, A. O. I., \& Shefrin, H. (2014). Technical analysis and individual investors. Journal of Economic Behavior \& Organization, 107, 487-511.

[29] Jiang, C., Ma, Y., \& An, Y. (2013). International portfolio selection with exchange rate risk: A behavioral portfolio theory perspective. Journal of Banking \& Finance, 37, 648-659.

[30] Kahneman, D., \& Tversky, A. (1979). Prospect theory: An analysis of decision under risk. Econometrica, 47, 263-292.

[31] Kennedy, J., \& Eberhart, R. C. (1995). Particle swarm optimization. In: Proceedings of the 1995 IEEE International Conference on Neural Networks, vol. 4, pp. 1942-1948. 
[32] Kennedy, J. (1997). The particle swarm: Social adaptation of knowledge. In: Proceedings of the 1997 IEEE International Conference on Evolutionary Computation, pp. 303-308.

[33] Kim, W. C., Kim, M. J., Kim, J. H., \& Fabozzi, F. J. (2014). Robust portfolios that do not tilt factor exposure. European Journal of Operational Research, 234, 411-421.

[34] Krohling, R. A., \& de Souza, T. T. M. (2012). Combining prospect theory and fuzzy numbers to multi-criteria decision making. Expert Systems with Applications, 39, 11487-11493.

[35] Liu, H. (2004). Optimal consumption and investment with transaction costs and multiple risky assets. The Journal of Finance, 59, 289-338.

[36] Liu, Y. J., Zhang, W. G., \& Xu, W. J. (2012). Fuzzy multi-period portfolio selection optimization models using multiple criteria. Automatica, 48, 3042-3053.

[37] Liu, Y., Fan, Z. P., \& Zhang, Y. (2014). Risk decision analysis in emergency response: A method based on cumulative prospect theory. Computers \& Operations Research, 42, 75-82.

[38] Liu, Y. J., \& Zhang, W. G. (2015). A multi-period fuzzy portfolio optimization model with minimum transaction lots. European Journal of Operational Research, 242, 933-941.

[39] Magron, C. (2014). Investors' aspirations and portfolio performance. Finance Research Letters, 11, 153-160.

[40] Maillet, B., Tokpavi, S., \& Vaucher, B. (2015). Global minimum variance portfolio optimisation under some model risk: A robust regression-based approach. European Journal of Operational Research, 244, 289-299.

[41] Markowitz, H. (1952). Portfolio selection. The Journal of Finance, 7, 77-91.

[42] Markowitz, H. (2014). Mean-variance approximations to expected utility. European Journal of Operational Research, 234, 346-355.

[43] Moon, Y., \& Yao, T. (2011). A robust mean absolute deviation model for portfolio optimization. Computers \& Operations Research, 38, 1251-1258.

[44] Pehlivanoglu, Y. V. (2013). A new particle swarm optimization method enhanced with a periodic mutation strategy and neural networks. IEEE Transactions on Evolutionary Computation, 17, 436-452.

[45] Runarsson, T. P., \& Yao, X. (2000). Stochastic ranking for constrained evolutionary optimization. IEEE Transactions on Evolutionary Computation, 4, 284-294.

[46] Shefrin, H., \& Statman, M. (2000). Behavioral portfolio theory. Journal of Financial and Quantitative Analysis, 35, 127-151.

[47] Silva, A., Neves, R., \& Horta, N. (2015). A hybrid approach to portfolio composition based on fundamental and technical indicators. Expert Systems with Applications, 42, 2036-2048.

[48] Soyster, A. L. (1973). Convex programming with set-inclusive constraints and applications to inexact linear programming. Operations Research, 21, 1154-1157.

[49] Takano, Y., \& Gotoh, J. (2014). Multi-period portfolio selection using kernel-based control 
policy with dimensionality reduction. Expert Systems with Application, 41, 3901-3914.

[50] Tiwana, A., Wang, J., Keil, M., \& Ahluwalia, P. (2007). The bounded rationality bias in managerial valuation of real options: Theory and evidence from IT projects. Decision Sciences, $38,157-181$.

[51] Tversky, A., \& Kahneman, D. (1992). Advances in prospect theory: Cumulative representation of uncertainty. Journal of Risk Uncertainty, 5, 297-323.

[52] Venkatraman, S., \& Yen, G. G. (2005). A generic framework for constrained optimization using genetic algorithm. IEEE Transactions on evolutionary computation, 9, 424-435.

[53] Wolpert, D. H., \& Macready, W. G. (1997). No free lunch theorems for optimization. IEEE Transactions on Evolutionary Computation, 1, 67-82.

[54] Zhu, S., Fan, M., \& Li, D. (2014). Portfolio management with robustness in both prediction and decision: A mixture model based learning approach. Journal of Economic Dynamics \& Control, $48,1-25$. 


\section{APPENDIX}

\section{Table 1: Comparison of different algorithms}

\begin{tabular}{cccccc}
\hline$\varepsilon$ & & PSO-1 & PSO-2 & PSO-3 & ALMV-PSO \\
\hline \multirow{2}{*}{0.05} & Mean & $-192,581.76$ & $-254,827.25$ & $-192,207.81$ & $\mathbf{- 1 9 0 , 2 7 9 . 3 6}$ \\
& Best & $-190,210.68$ & $-194,726.24$ & $-189,995.32$ & $\mathbf{- 1 8 9 , 2 9 2 . 0 9}$ \\
& Time $(\mathrm{s})$ & 38.19 & $\mathbf{1 5 . 5 3}$ & 44.13 & 48.29 \\
\hline \multirow{3}{*}{0.10} & Mean & $-135,722.99$ & $-201,386.15$ & $-135,273.49$ & $\mathbf{- 1 3 2 , 3 3 3 . 8 5}$ \\
& Best & $-132,254.11$ & $-141,278.99$ & $-131,473.49$ & $\mathbf{- 1 2 8 , 5 4 5 . 6 3}$ \\
& Time (s) & 33.93 & $\mathbf{1 6 . 5 2}$ & 37.11 & 45.96 \\
\hline \multirow{2}{*}{0.15} & Mean & $-87,928.84$ & $-133,064.95$ & $-87,688.95$ & $\mathbf{- 8 5 , 5 3 0 . 3 3}$ \\
& Best & $-84,373.11$ & $-88,952.73$ & $-83,134.59$ & $\mathbf{- 8 2 , 0 9 7 . 9 3}$ \\
& Time (s) & 34.67 & $\mathbf{3 2 . 2 8}$ & 38.92 & 44.91 \\
\hline \multirow{2}{*}{0.20} & Mean & $-46,020.72$ & $-107,895.71$ & $-45,965.19$ & $\mathbf{- 3 9 , 3 7 0 . 4 4}$ \\
& Best & $-42,390.96$ & $-55,095.03$ & $-42,252.82$ & $\mathbf{- 4 1 , 9 4 8 . 9 3}$ \\
& Time (s) & 38.03 & $\mathbf{2 3 . 1 7}$ & 39.31 & 47.02 \\
\hline
\end{tabular}

Note: The table presents the computational result of PSO-1, PSO-2, PSO-3 and ALMV-PSO. All algorithms are run 10 times independently. The best results among the algorithms are shown in bold.

\section{Table 2: Robustness among Different Portfolios}

\begin{tabular}{lccccc}
\hline \multirow{2}{*}{ Portfolio Model } & \multicolumn{4}{c}{ Robust Portfolio } & \multirow{2}{*}{ Nominal Portfolio } \\
\cline { 2 - 5 } & $\varepsilon=0.05$ & $\varepsilon=0.10$ & $\varepsilon=0.15$ & $\varepsilon=0.2$ & \\
\hline Robust Measure & $681,449.18$ & $692,839.18$ & $705,361.11$ & $736,600.37$ & $1,197,378.13$ \\
\hline
\end{tabular}

Note: The ALMV-PSO algorithm is run separately 10 times, and the best value is selected to stand for the portfolio performance. Following the actual action, we solve the robustness measure at each period. In order to reflect the robustness level over the entire investment, the robustness measure of each period is added up to represent robustness of the portfolio model. Interested readers can refer to Kim et al. (2014) for a detail discussion about the robustness measure. 


\section{Table 3: Actual and robust wealth of portfolios}

\begin{tabular}{cccccc}
\hline Investment duration & & $\varepsilon=0.05$ & $\varepsilon=0.10$ & $\varepsilon=0.15$ & $\varepsilon=0.20$ \\
\hline \multirow{2}{*}{$1 / 2014 \sim 3 / 2014$} & Actual wealth & $1,059,351.67$ & $1,059,661.71$ & $1,060,069.07$ & $1,059,827.36$ \\
& Robust wealth & $973,456.45$ & $983,573.63$ & $990,897.23$ & $997,705.18$ \\
\hline \multirow{2}{*}{$4 / 2014 \sim 6 / 2014$} & Actual wealth & $1,102,259.03$ & $1,102,570.77$ & $1,103,064.98$ & $1,102,907.43$ \\
& Robust wealth & $1,016,216.16$ & $1,026,083.34$ & $1,034,025.36$ & $1,043,669.28$ \\
\hline $7 / 2014 \sim 9 / 2014$ & Actual wealth & $1,129,030.28$ & $1,129,369.16$ & $1,129,743.35$ & $1,129,858.62$ \\
& Robust wealth & $1,048,665.81$ & $1,061,457.44$ & $1,071,070.24$ & $1,080,509.68$ \\
\hline $10 / 2014 \sim 12 / 20144$ & Actual wealth & $1,146,972.25$ & $1,148,758.41$ & $1,150,411.31$ & $1,153,514.66$ \\
& Robust wealth & $1,110,498.87$ & $1,122,043.84$ & $1,130,292.49$ & $1,137,514.66$ \\
\hline
\end{tabular}

Note: The ALMV-PSO algorithm is run separately 10 times, and the best value is selected to stand for the portfolio performance. The actual wealth is calculated by adding up the actual wealth of assets at each period. The robust wealth is represented by $W_{t}^{R}$ at Eq. (5). 
Figure 1: Multi-period investment

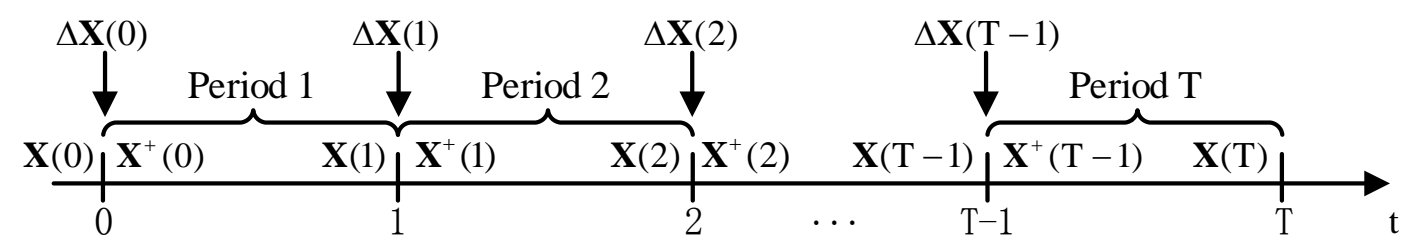

Note: The graph shows the multi-period investment procedure. Suppose at time $t$, an investor holds the portfolio $\mathbf{X}(t), t=0, \ldots, T$. The investor could dynamically adjust the portfolio at the end of each period based on the realized return and updated information about the security market. Let $\Delta \mathbf{X}(t)$ be the adjustment of portfolio at time $t, t=0, \ldots, T-1$. After the adjustment at time $t$, we could obtain the adjusted portfolio $\mathbf{X}^{+}(t)=\mathbf{X}(t)+\Delta \mathbf{X}(t)$, $t=0, \ldots, T-1$.

\section{Figure 2: Pseudocode of stochastic ranking approach}

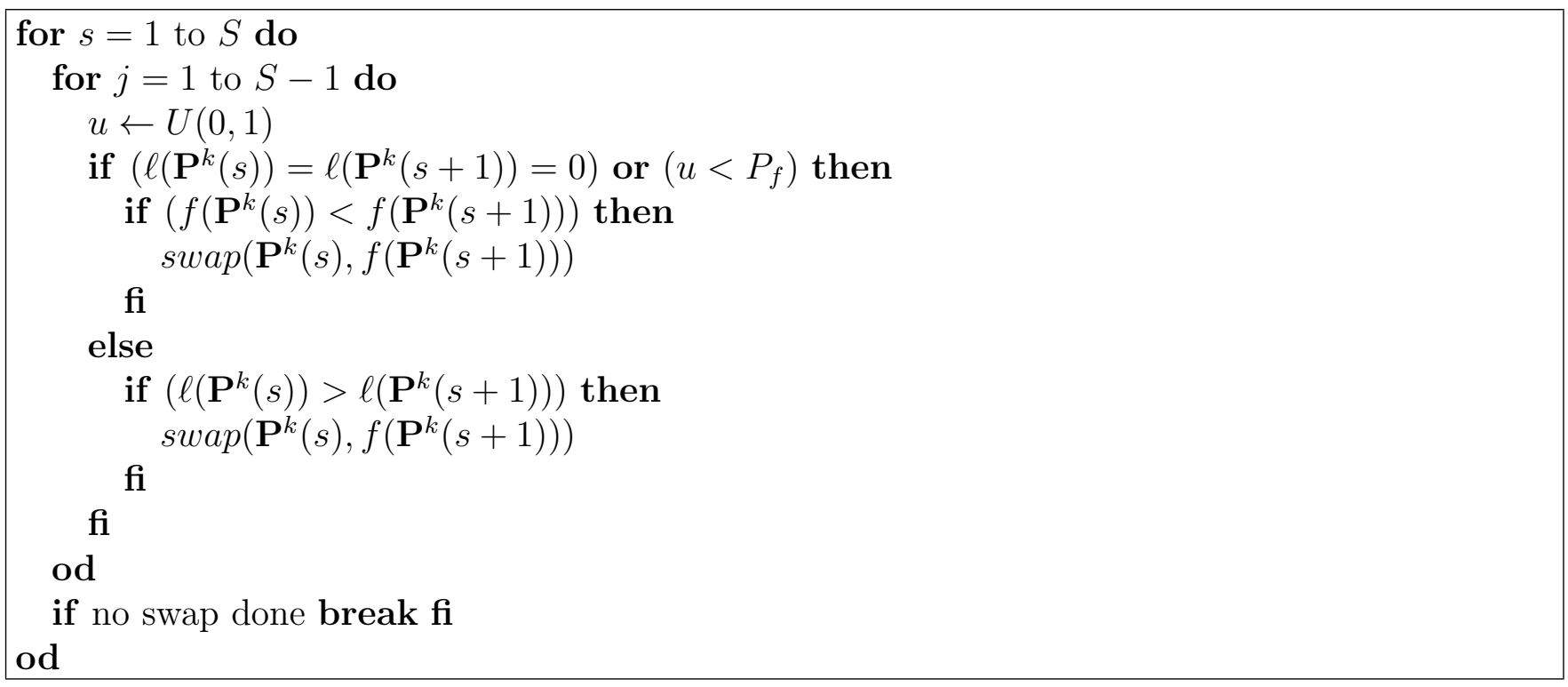

Note: Stochastic ranking approach using a bubble-sort-like procedure where $U(0,1)$ is a uniform random number generator. When $P_{f}=0$ the ranking is an over-penalization and for $P_{f}=1$ the ranking is an under-penalization (Runarsson and Yao, 2000). 
Figure 3: Pseudocode of the improved stochastic ranking approach for updating pbest

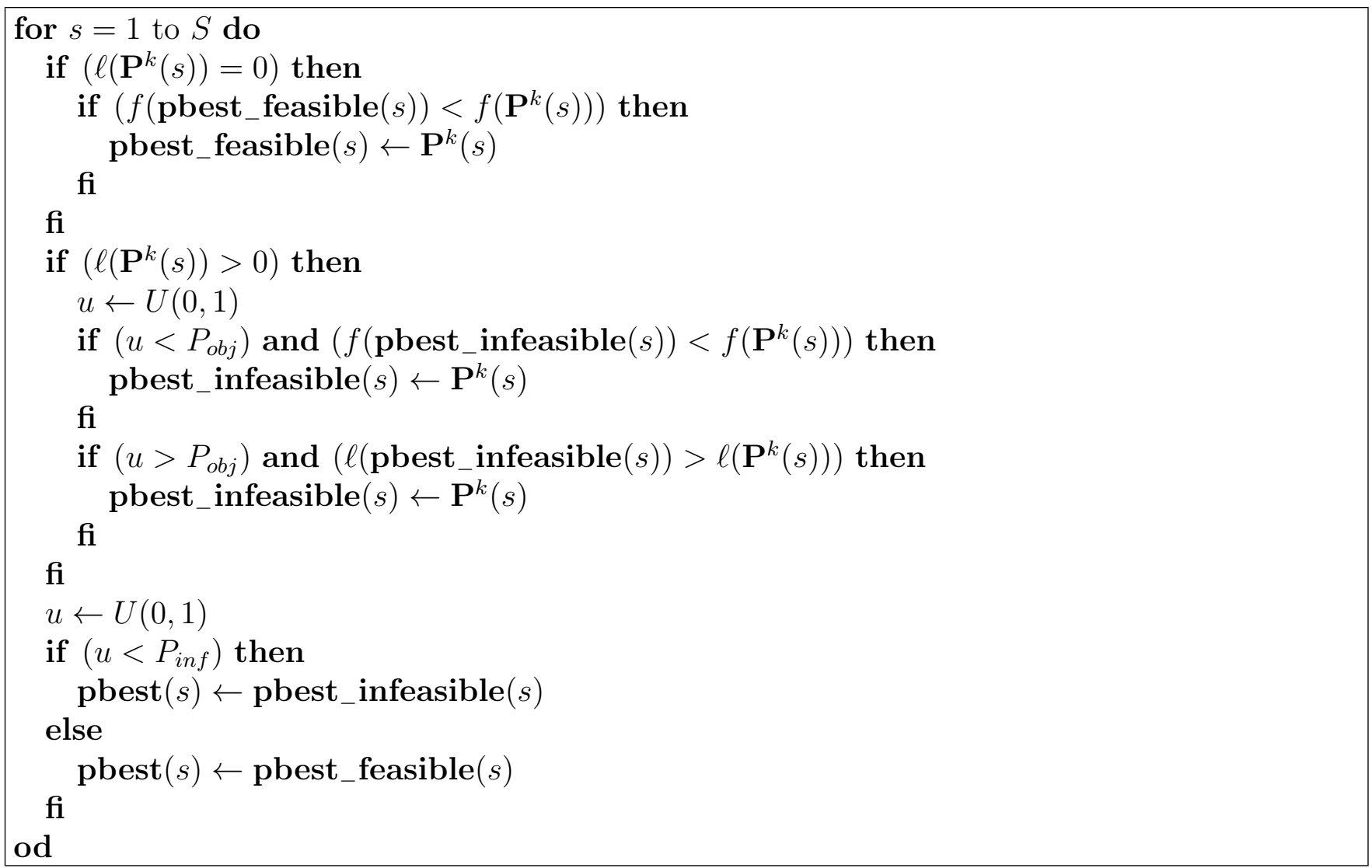

Note: Inspired by the stochastic ranking approach (Runarsson and Yao, 2000), we design an improved stochastic ranking approach for PSO algorithm. While the individual is infeasible, the probability of comparing it according to the objective function is $P_{o b j}$. Next, the algorithm could choose an infeasible individual as the pbest with the probability $P_{\text {inf }}$. pbest_feasible $(s)$ denotes the personal best of particle $s$ in feasible region. pbest_infeasible $(s)$ denotes the personal best of particle $s$ in infeasible region. 
Figure 4: Pseudocode of the improved stochastic ranking approach for updating gbest

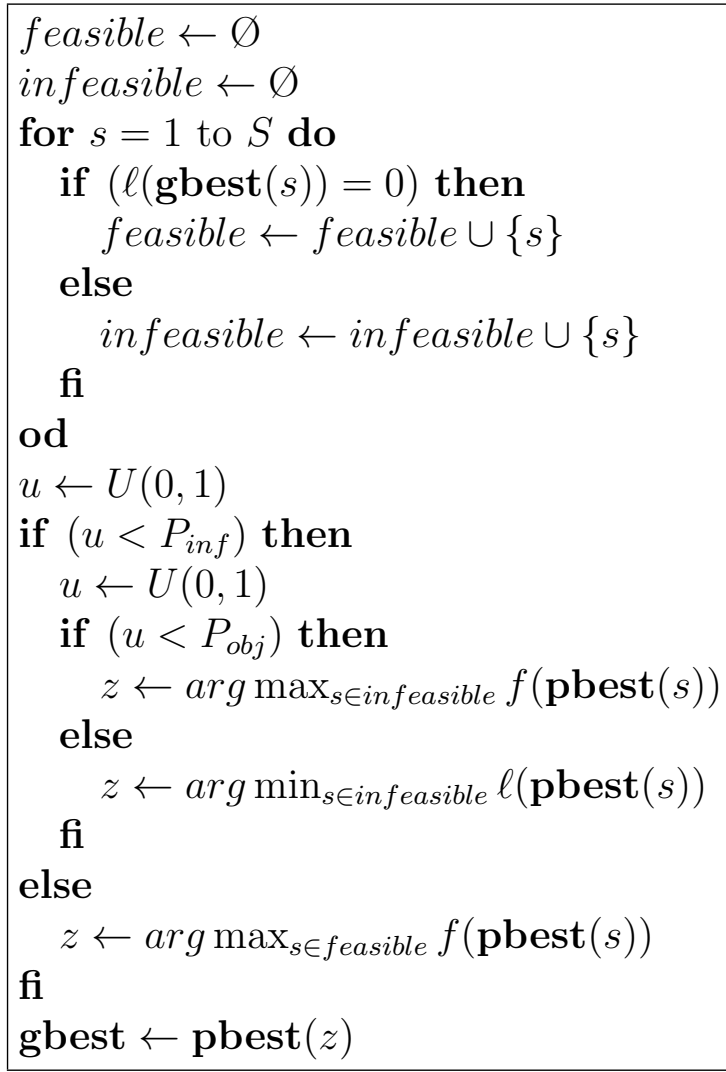

Note: In order to select a gbest from $\operatorname{pbest}(s), s=1, \ldots, S$, we divide the pbest $(s), s=1, \ldots, S$ into two parts. The feasible $\operatorname{pbest}(s)$ transfers into feasible, while the infeasible pbest $(s)$ transfers into infeasible, $s=1, \ldots, S$. For the infeasible pbest $(s)$, the probability of comparing them according to the objective function is $P_{o b j}$. Next, the algorithm could choose an infeasible individual as the gbest with the probability $P_{\text {inf }}$. $\varnothing$ denotes an empty set. 


\section{Figure 5: Lifespan control operator}

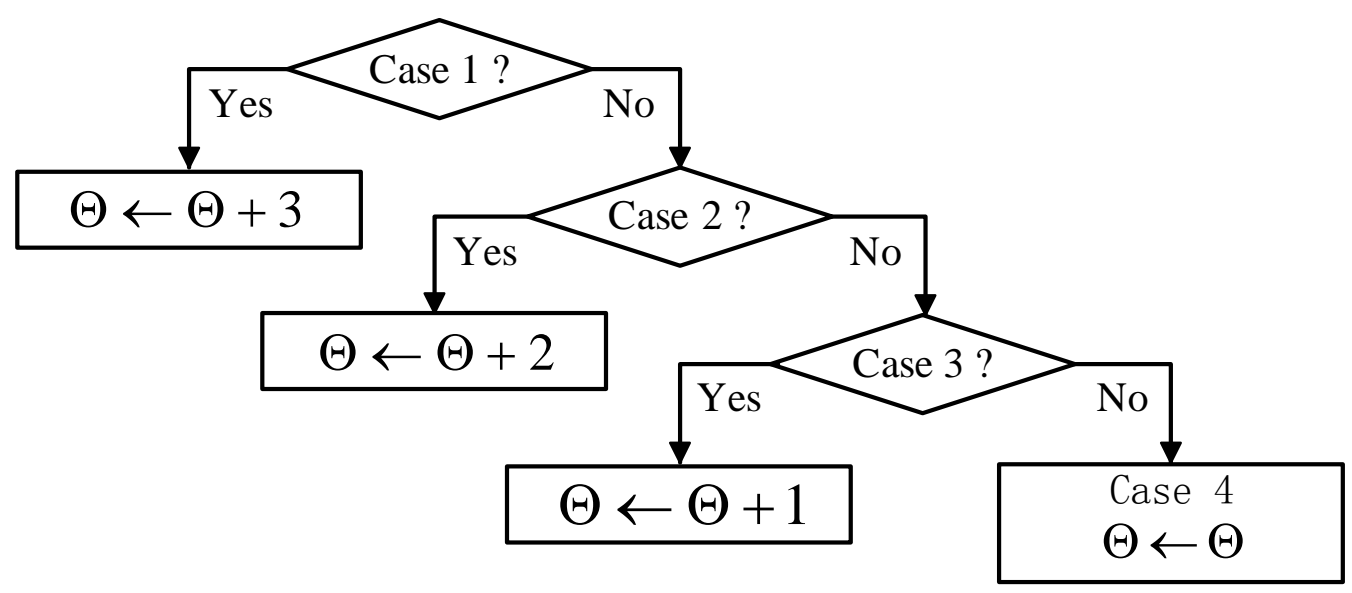

Note: Following the method of Chen et al. (2013), we uses a lifespan control operator to adjust the leader's lifespan. While the leader has a good leading power (Case 1), the lifespan $\Theta$ of the leader increased by 3. While the leader has a fair leading power (Case 2), the lifespan $\Theta$ of the leader increased by 2. While the leader has a poor leading power (Case 3), the lifespan $\Theta$ of the leader increased by 1 . While the leader has a no leading power (Case 4), the lifespan $\Theta$ of the leader remains unchanged. 